\title{
Relationship Between Strength of Hip Muscles and Performance of Close Kinetic Chain Dynamic Lower Extremity Stability Test
}

\author{
Hyeon-ju Lee1 ${ }^{1}$ BPT, PT, Ui-jae Hwang ${ }^{2,3}$, PhD, PT, Sung-hoon Jung ${ }^{2,3}$, PhD, PT, Sun-hee Ahn ${ }^{2,3}$, PhD, PT, \\ Oh-yun Kwon ${ }^{2,3}$, PhD, PT \\ ${ }^{1}$ Department of Physical Therapy, The Graduate School, Yonsei University, ${ }^{2}$ Department of Physical Therapy, College of Health Science, \\ Yonsei University, ${ }^{3}$ Kinetic Ergocise Based on Movement Analysis Laboratory, Wonju, Korea
}

\author{
Article Info \\ Received October 8, 2020 \\ Revised October 27, 2020 \\ Accepted October 27, 2020 \\ Corresponding Author \\ Oh-yun Kwon \\ E-mail: kwonoy@yonsei.ac.kr \\ https://orcid.org/0000-0002-9699-768X
}

\section{Key Words}

Hip

Lower extremity

Muscle strength
Background: The hip muscle plays various roles. Several types of functional performance tests are used for the assessment of patients with various lower extremity injuries. Hip muscle functions are important to test the performance of maintaining the spine, pelvic, and leg during bridging exercise. We designed a novel functional performance test tool, which we named close kinetic chain dynamic lower extremity stability (CKCLE) test to assess hip muscle functions.

Objects: The purpose of this study was to determine the relationship between CKCLE test and hip extensor, external rotator, and abductor strengths.

Methods: Twenty-two subjects were recruited in the present study (13 males and 9 females). The hip extensor, external rotator, and abductor muscle strengths were measured using a Smart KEMA strength sensor. When the examiner said "Go", the subject performed the CKCLE test by moving one leg from the floor and touching the opposite knee and then return to the floor while maintaining the bridging position. The subjects attempted as many "touches" as possible in the allotted time (20 seconds) during the maximal tests. The correlation between the hip muscle (extensor, external rotator, and abductor) strength of the supporting leg and the number of CKCLE tests performed in 20 seconds was determined using the Pearson correlation.

Results: Hip extensor $(r=0.626, p<0.05)$, hip external rotator $(r=0.616, p<0.05)$, and hip abductor muscle strengths $(r=0.475, p<0.05)$ positively correlated with the number of CKCLE tests performed.

Conclusion: We designed a CKCLE test and found that performance in the test correlated with hip extensor, external rotator, and abductor muscle strengths. The result suggests that the CKCLE test can be applied as a performance test to assess the functions of the hip extensor, external rotator, and hip abductor muscles.

\section{INTRODUCTION}

The hip muscle plays various roles; it helps control pelvic stability and leg alignment in the frontal, sagittal, and transverse planes [1]. Hip muscle strength and endurance play a significant role in injury prevention, normalizing gait patterns and posture, eliminating pain, and enhancing athletic performance [2]. Impaired hip muscle strength has been associated with various musculoskeletal disorders, such as lower back and pelvic pain, patellofemoral pain, and iliotibial band syndrome [3]. Thus, it is very important to evaluate hip muscle function to prevent musculoskeletal disorders caused due to the impairment of hip muscles.

Manual muscle strength testing is commonly used for the measure the strength of hip muscles [4]. Contrary to traditional examination, measurements such as range of motion (ROM) and strength tests are performed; functional performance tests examine how multiple body segments and systems interact [5]. Several types of functional performance tests are used to assess patients with various lower extremity injuries. These may be categorized into movement, hop/jump, balance, and agility tests [6]. 
The star excursion balance test (SEBT), the Y-balance test, single-leg squat, and single-leg stance were used to test the lower extremity function. In addition, a single-leg hamstring bridge (SLHB) test is used to predict hamstring injury in sports players. The SLHB test is a simple clinical test for assessing the hamstring strength using repetitive hip and knee movements [7]. Although there is the SLHB test for the measurement of the hamstring strength, a simple clinical test for assessing hip muscles strength is needed. This is because, the hip muscles play an important role in forming a kinetic chain by connecting the lower extremities, the thigh and the pelvis. Thus, testing the hip muscle function is important to test the performance of the spine, pelvis, and leg during bridging exercises.

In the upper extremity, there is a closed kinetic chain upper extremity stability (CKCUES) test that evaluates the quantitative data (score) for an upper-extremity task in a closed kinetic chain within time [8]. However, for assessing hip muscle function, there is no performance test that evaluates data on lower limb movements in a closed kinetic chain within time, as in the CKCUES test. Thus, we designed a novel functional performance test tool, which we named close kinetic chain dynamic lower extremity stability (CKCLE) test to assess hip muscle function. The single-leg bridge exercise has been used to improve hip muscle function [9]. A test was devised to count the number of repetitively performed motions with opposite side hip external rotation, adduction, and extension for touching the supporting knee with the moving leg heel while maintaining the single-leg bridge position for 20 seconds. The CKCUES test had an evaluation time of 15 seconds [8], but it was considered that lower extremity muscles are more powerful than upper extremity muscles. In addition, when 30 seconds was used to evaluate the CKCLE test time, subjects felt the muscle fatigue at the first CKCLE test. Considering these reasons, the CKCLE test time was set to 20 seconds. Therefore, the purpose of this study was to determine the relationship between performance of CKCLE test and strengths of hip extensor, external rotator, and abductor muscle.

\section{MATERIALS AND METHODS}

\section{Subjects}

We used the $G^{*}$ power program to determine the sample size using the pilot data. The necessary sample size of 22 was calculated from pilot study data of 8 subjects, with a power of 0.80 , alpha level of 0.05 , and effect size of 0.838 . Therefore, 22 volunteers participated in this study (13 males and 9 females). To be included in the study, subjects had to meet the following criteria: 1) no prior history of unresolved pain, injury, or surgery to either hip, knee, or ankle; and 2) currently not taking any prescribed medication for pain [10]. The study procedures were explained prior to testing, and each subject provided informed consent. The study was approved by the Yonsei University Mirae Institutional Review Board (approval No. 1041849-202002-BM-023-01).

\section{Measurement of Hip Muscle Strength}

The hip extensor, external rotator, and abductor muscle strengths were measured using a Smart KEMA strength sensor (KOREATECH Co. Ltd., Seoul, Korea) (Figure 1). The tension gauge was $3 \mathrm{~kg}$ in the starting position to control the tension of the belt in each test position. Isometric strength measurements at both legs were performed twice for $5 \mathrm{~s}$ under three conditions: (1) hip extension, (2) hip external rotation, and (3) hip abduction. The force signals were measured and transferred to an electronic tablet device (Galaxy Tab A6 10.1; Samsung Inc., Seoul, Korea) via Bluetooth device connection and analyzed with the Smart KEMA application software (KOREATECH Co. Ltd.). The value was calculated as the average of the values for 3 seconds within the 5 seconds. The mean values of the two trials were used for data analysis.

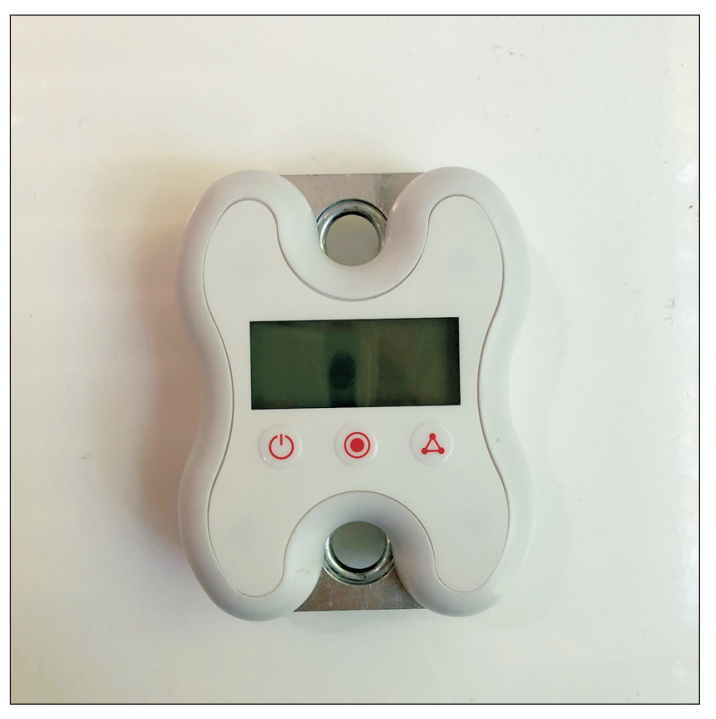

Figure 1. Smart KEMA strength sensor. 


\section{1) Hip extensor muscle strength}

The strength of the hip extensor muscle was measured in the prone position with $0^{\circ}$ hip flexion and $90^{\circ}$ knee flexion. A strap was attached to the distal femur of the subjects. The tension of the belt was adjusted for each subject with hip extension at $0^{\circ}$ (Figure 2). To measure the maximum isometric force of hip extension, subjects were instructed to extend their hip and pull the line with maximum effort, without lumbar extension for 5 seconds.

\section{2) Hip external rotator muscle strength}

The strength of the hip external rotator muscle was measured in the side-lying position with $0^{\circ}$ hip flexion and $90^{\circ}$ knee flexion. An orthopedic belt was attached to a steel bar on the therapeutic table, and a strap was placed at the ankle (Figure 3). To measure the maximum force of the hip external rotator muscle, subjects were instructed to rotate the hip externally with maximum effort without pelvic motion for $5 \mathrm{sec}^{-}$ onds.

\section{3) Hip abductor muscle strength}

The strength of the hip abduction muscle was measured in the supine position with $0^{\circ}$ hip abduction. A strap was attached to the ankle. The tension of the belt was adjusted for each subject with hip abduction at $0^{\circ}$ (Figure 4). To measure the maximum isometric force of hip abduction, subjects were instructed to abduct their hip and pull the line with maximum effort for 5 seconds without hip flexion and knee flexion.

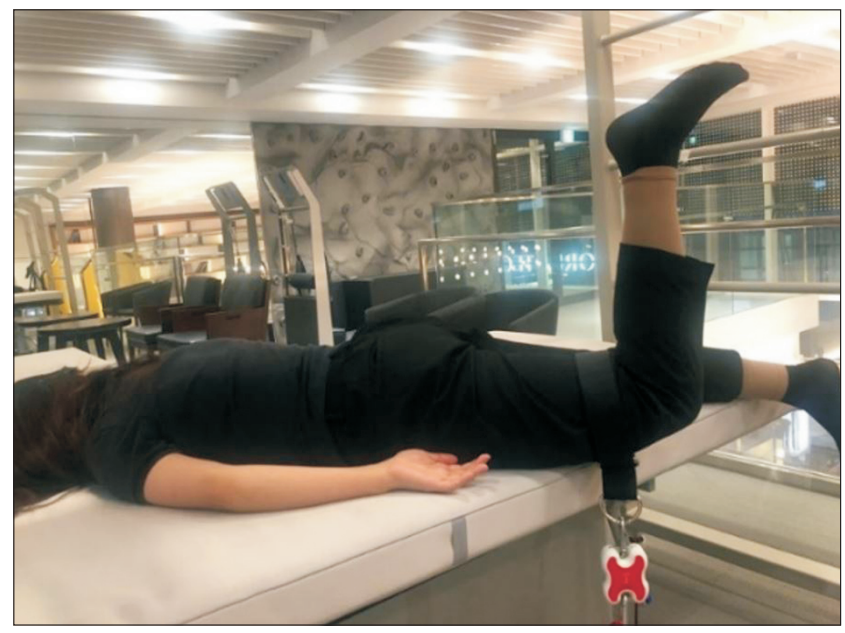

Figure 2. Strength of hip extensor muscle.

\section{Measurement Using the Close Kinetic Chain Dynamic Lower Extremity Stability Test}

To begin, the subjects assumed a bridge position, with the knee flexed $135^{\circ}$. The feet were positioned directly over the knees (Figure 5A). When the examiner said "go," the subjects performed the CKCLE test by moving one leg from the floor, touching the opposite knee as in performing hip external rotation, adduction, and extension (Figure 5B); then replacing the leg on the floor while maintaining the bridging position (Figure 5C). A single test consisted of continuing this alternating procedure for 20 seconds. Subjects attempted as many "touches" as possible in the allotted time during the maximal tests. One cycle of touch was defined as "when the heel of the moving leg touched the opposite knee and returned to the ground while maintaining the bridging position." The target bar was used

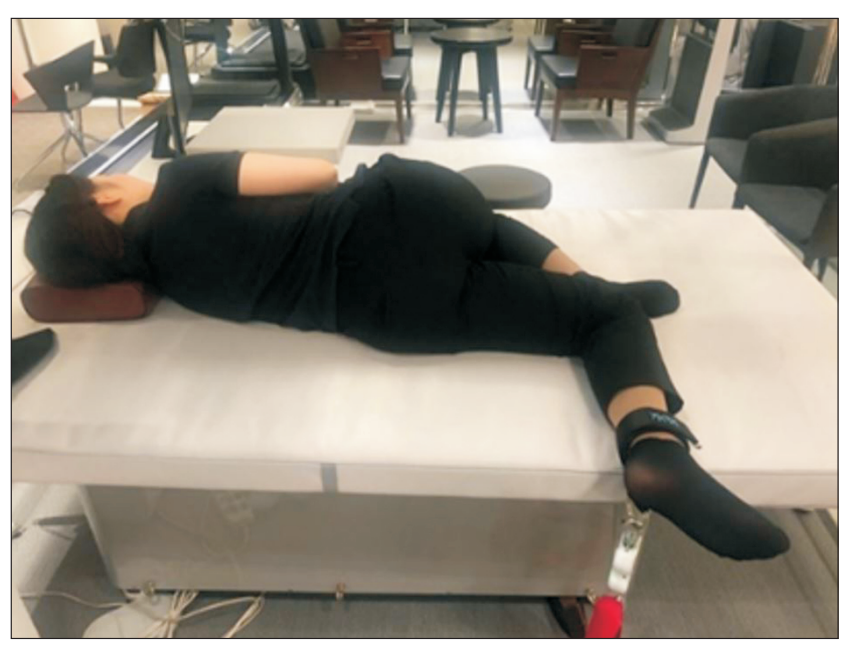

Figure 3. Strength of hip external rotator muscle.

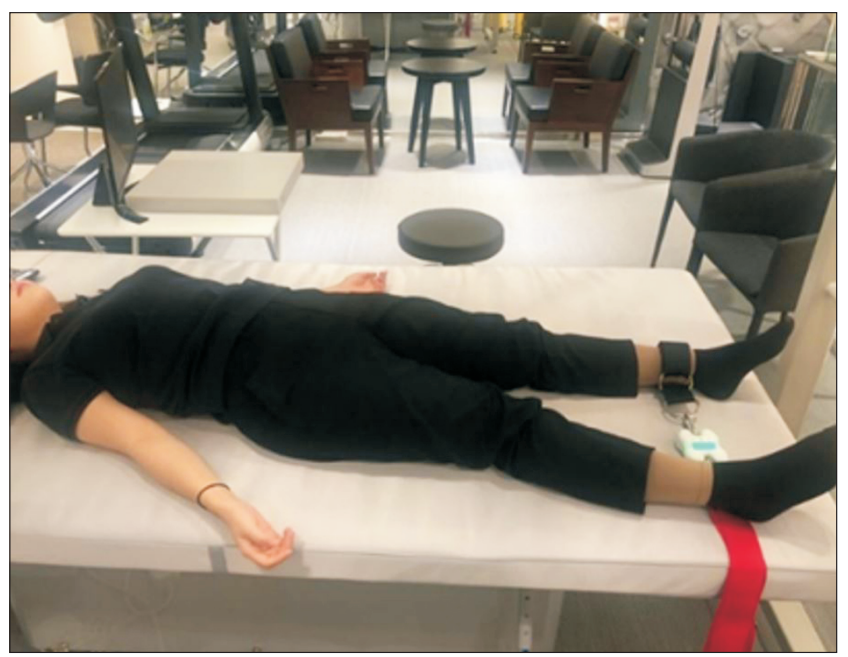

Figure 4. Strength of hip abductor muscle. 

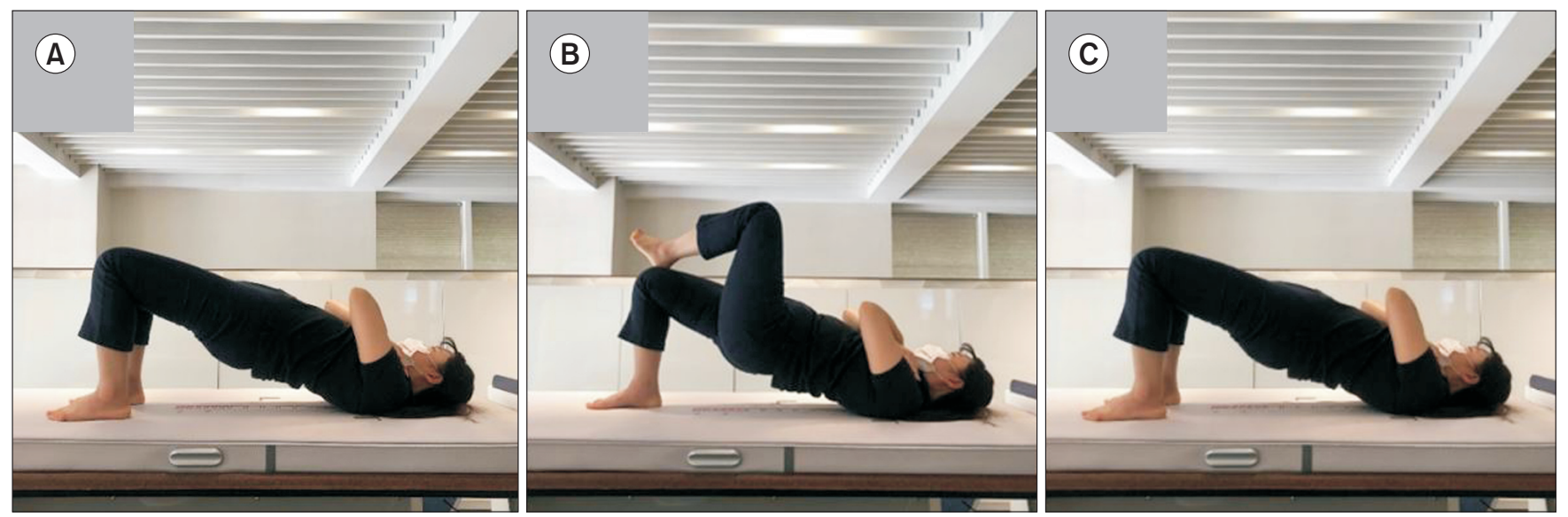

Figure 5. (A-C) Close kinetic chain dynamic lower extremity stability test.

to equally control the position of the pelvis, not to drop their buttocks on the floor during the test. Continuous feedback was provided not to drop the buttocks to the floor during the CKCLE test. The caution was given if the pelvic dropped from the target bar, and the test was stopped when the pelvic was not at the target bar despite the caution. Before the test, the subjects had sufficient practice. The examiner counted the number of repeated movements during the test.

\section{Procedures}

This study was conducted in a laboratory setting. Muscle strengths of all subjects were measured at one visit, and two trials of each measurement were performed, with a 60 seconds rest period between each trial and a 3 minutes rest period between each measurement. Before the CKCLE test was performed, subjects were familiarized with the test pattern for a period of approximately 5 minutes to achieve a proper test capability. The subjects performed two trials with each leg and had a 5 minutes rest period between each trial. The four measurements were tested in a random order as determined by a website-based application (http://www.randomization.com).

\section{Statistical Analysis}

The data were analyzed using PASW version 18.0 (IBM Co., Armonk, NY, USA) for Windows. The Kolmogorov-Smirnov test was used to verify that the data were normally distributed. Intra-class correlation coefficients (ICCs) with 95\% confidence intervals (CIs) were used to determine the intra-reliability of the Smart KEMA strength sensor and CKCLE test. The correlation between the hip muscles (extensor, external rotator, and abductor) strengths of the supporting leg and performance
Table 1. Characteristics of the subjects

\begin{tabular}{lccc}
\hline Characteristic & Total $(\mathrm{N}=22)$ & Male $(\mathrm{n}=13)$ & Female $(\mathrm{n}=9)$ \\
\hline Age $(\mathrm{yr})$ & $29.9 \pm 3.9$ & $29.1 \pm 2.9$ & $31.5 \pm 5$ \\
Body weight $(\mathrm{kg})$ & $66.9 \pm 12.1$ & $75.2 \pm 6.7$ & $54.5 \pm 6.8$ \\
Height $(\mathrm{cm})$ & $169.8 \pm 10.1$ & $176.6 \pm 5.8$ & $160.1 \pm 5.4$ \\
\hline
\end{tabular}

Values are presented as mean \pm standard deviation.

of CKCLE tests was determine using the Pearson correlation. Mukaka [11] suggested threshold values of $0.0-0.3,0.3-0.5$, $0.5-0.7,0.7-0.9$, and $0.9-1.0$ for negligible, low, moderate, high, and very high correlation coefficients, respectively. In all analyses, $p<0.05$ was considered to statistically significant.

\section{RESULTS}

General characteristics of the study subjects are listed in Table 1 . The data collected for all variables were normally distributed. The ICCs for measuring hip extensor muscle strength was 0.979 (95\% CIs: 0.961-0.988), for hip external rotator muscle strength was 0.976 (95\% CIs: 0.955-0.987), and for hip abductor muscle strength was 0.950 (95\% CIs: 0.907-0.974). In addition, the ICCs for the CKCLE test were 0.820 (95\% CIs: 0.621-0.914). Table 2 shows the mean and standard deviation of the number of CKCLE tests and strength of hip muscles. Table 3 shows the Pearson's correlation coefficients between the CKCLE test and the hip extensor, the external rotator, and the abductor muscle strengths. Hip extensor $(r=0.626, p<$ $0.05)$, hip external rotator $(r=0.616, p<0.05)$, and hip abductor muscle strengths $(r=0.475, p<0.05)$ positively correlated with the number of CKCLE tests performed. 
Table 2. CKCLE test, strength of hip muscles

\begin{tabular}{llll}
\hline \multicolumn{1}{c}{ Variable } & Total $(\mathrm{N}=22)$ & Male $(\mathrm{n}=13)$ & Female $(\mathrm{n}=9)$ \\
\hline CKCLE test (number) & $22.36 \pm 4.37$ & $24.77 \pm 3.52$ & $21.33 \pm 4.75$ \\
Hip extensor muscle $(\mathrm{kg})$ & $33.46 \pm 10.86$ & $37.02 \pm 10.63$ & $28.31 \pm 9.18$ \\
Hip external rotator muscle $(\mathrm{kg})$ & $16.71 \pm 5.16$ & $18.94 \pm 4.72$ & $13.50 \pm 4.00$ \\
Hip abductor muscle $(\mathrm{kg})$ & $17.28 \pm 4.45$ & $18.99 \pm 4.37$ & $14.80 \pm 3.33$ \\
\hline
\end{tabular}

Values are presented as mean \pm standard deviation. CKCLE, close kinetic chain dynamic lower extremity stability.

Table 3. Pearson's correlation coefficients between the CKCLE test and the hip muscles strength

\begin{tabular}{lllll}
\hline \multicolumn{1}{c}{ Variable } & CKCLE test & Hip extensor & Hip external rotator & Hip abductor \\
\hline CKCLE test & 1.0 & & & \\
Hip extensor & $0.626^{* *}$ & 1.0 & 1.0 & $0.569^{* *}$ \\
Hip external rotator & $0.616^{* *}$ & $0.531^{* *}$ & 1.0 \\
Hip abductor & $0.475^{* *}$ & $0.470^{* *}$ & 1.0 \\
\hline
\end{tabular}

CKCLE, close kinetic chain dynamic lower extremity stability. ${ }^{* *} p<0.05$.

\section{DISCUSSION}

We investigated the relationship between the CKCLE test and hip extensor, external rotator and abductor muscle strengths. The present study measured the strengths of three hip muscles, which control pelvic alignment during a single-leg bridge exercise, such as the hip extensor, external rotator, and abductor. The repetition number of the CKCLE test was positively correlated with hip extensor, external rotator and hip abductor muscle strengths. The results showed a moderate correlation between the CKCLE test and hip extensor and external rotator muscle strengths, although the recruitment of back extensor, lumbopelvic stability, and knee control may affect performance during the CKCLE test. Our findings indicate that the CKCLE test can be applied to measure the function of the hip extensor, external rotator, and hip abductor muscles, when hip muscle strength cannot be measured in a clinical setting.

The reason of the positive correlation between the strength of hip extensor muscle and performance of CKCLE test is as follows: CKCLE test was designed based on the single-leg bridge exercise. The single-leg bridge exercise activated $51 \%$ of gluteus maximus maximal voluntary isometric contraction in a previous study [2]. In addition, we controlled the position in knee flexion at $135^{\circ}$. A previous study suggested that position in knee flexion $135^{\circ}$ during one leg bridge could facilitate the gluteus maximus more than hamstring muscles [2]. Although we did not measure activity of the gluteus maximus and hamstring, it is possible that gluteus maximus may be more recruited than hamstring while assessing the CKCLE test.
The hip external rotator muscle strength showed a moderate correlation with the CKCLE test. The correlation between the hip external rotator muscle strength and the CKCLE test could be explained by the recruitment of the hip external rotators, such as the posterior part of gluteus medius and piriformis, are necessary to maintain pelvic stability and a hip neutral position during the CKCLE test. This could affect the correlation between strength of hip external rotators and the performance of CKCLE tests. Hip external rotator muscle weakness can cause excessive internal hip rotation and foot pronation, and increases the angle of knee valgus during weight-bearing activities [12]. Thus, the CKCLE test could be applied to measure the hip external rotator muscle function in clinical settings.

There was a significant positive correlation between strength of hip abductors and performance of CKCLE test, although the correlation was less than other muscles. The reason for the correlation between the hip abductor muscle strength and the CKCLE test is the pelvic control that is required to maintain minimized pelvic movement during the CKCLE test. Similar to the hip external rotator muscle as an anatomical overlapped structure, recruitment of the hip abductor muscle would also have been needed to maintain pelvic stability during the $\mathrm{CK}$ CLE test.

For another lower extremity functional tests, the SEBT and the Y-balance tests have been used frequently to assess dynamic postural control with unilateral weight-bearing conditions. Hubbard et al. [13], investigated that hip extensor muscle strength correlated with the posterior-medial $(r=0.48, p<0.05)$ and posterior-lateral areas $(r=0.49, p<0.05)$. Other studies 
have shown that ankle dorsiflexion, knee flexion, and hip flexion ROM were associated with Y-balance test and SEBT numbers $[6,14,15]$. Thus, although it is difficult to directly compare with other tests, the CKCLE test could be more suitable for selectively evaluating the function of hip muscle strength than other measurement tools.

This study has several limitations. The test was not evaluated to patients or various age groups. Therefore, this result cannot be generalized to all subjects. In addition, it is necessary to compare the performance of CKCLE test in subjects with normal and weak strength of hip muscle, and to determine whether performance of the CKCLE test improves after strengthening the hip extensor and external rotator muscles.

\section{CONCLUSIONS}

We designed a CKCLE test and found that performance in the test correlated with hip extensor, external rotator, and abductor muscle strengths. The results suggest that the CKCLE test can be applied as a performance test to assess the functions of the hip extensor, external rotator, and hip abductor muscles.

\section{ACKNOWLEDGEMENTS}

This study was supported by the "Brain Korea 21 FOUR Project", the Korean Research Foundation for Department of Physical Therapy in the Graduate School of Yonsei University.

\section{CONFLICTS OF INTEREST}

No potential conflict of interest relevant to this article was reported.

\section{AUTHOR CONTRIBUTION}

Conceptualization: HL, UH, SJ, SA, OK. Data curation: HL, UH, SJ, SA. Formal analysis: HL, UH, SJ, SA. Investigation: HL, UH, SJ, SA. Methodology: HL, UH, SJ, SA, OK. Project administration: HL, UH, SJ, SA. Resources: UH, SJ, SA, OK. Validation: UH, SJ, OK. Visualization: UH. Writing - original draft: HL, UH, SJ, SA. Writing - review \& editing: HL, UH, SJ, SA, OK.

\section{ORCID}

Hyeon-ju Lee, https://orcid.org/0000-0003-4169-6836

Ui-jae Hwang, https://orcid.org/0000-0002-2050-5503

Sung-hoon Jung, https://orcid.org/0000-0002-3704-1514

Sun-hee Ahn, https://orcid.org/0000-0001-7660-8598

\section{REFERENCES}

1. Cichanowski HR, Schmitt JS, Johnson RJ, Niemuth PE. Hip strength in collegiate female athletes with patellofemoral pain. Med Sci Sports Exerc 2007;39(8):1227-32.

2. Lehecka BJ, Edwards M, Haverkamp R, Martin L, Porter K, Thach K, et al. Building a better gluteal bridge: electromyographic analysis of hip muscle activity during modified singleleg bridges. Int J Sports Phys Ther 2017;12(4):543-9.

3. Ferber R, Noehren B, Hamill J, Davis IS. Competitive female runners with a history of iliotibial band syndrome demonstrate atypical hip and knee kinematics. J Orthop Sports Phys Ther 2010;40(2):52-8.

4. Augustsson J, Thomeé R. Ability of closed and open kinetic chain tests of muscular strength to assess functional performance. Scand J Med Sci Sports 2000;10(3):164-8.

5. Kivlan BR, Carcia CR, Clemente FR, Phelps AL, Martin RL. Reliability and validity of functional performance tests in dancers with hip dysfunction. Int J Sports Phys Ther 2013;8(4):3609.

6. Kivlan BR, Martin RL. Functional performance testing of the hip in athletes: a systematic review for reliability and validity. Int J Sports Phys Ther 2012;7(4):402-12.

7. Freckleton G, Cook J, Pizzari T. The predictive validity of a single leg bridge test for hamstring injuries in Australian Rules Football Players. Br J Sports Med 2014;48(8):713-7.

8. Goldbeck TG, Davies GJ. Test-retest reliability of the closed kinetic chain upper extremity stability test: a clinical field test. J Sport Rehabil 2000;9(1):35-45.

9. Selkowitz DM, Beneck GJ, Powers CM. Which exercises target the gluteal muscles while minimizing activation of the tensor fascia lata? Electromyographic assessment using fine-wire electrodes. J Orthop Sports Phys Ther 2013;43(2):54-64.

10. Greenberger HB, Paterno MV. Relationship of knee extensor strength and hopping test performance in the assessment of lower extremity function. J Orthop Sports Phys Ther 1995;22(5):202-6. 
11. Mukaka MM. A guide to appropriate use of Correlation coefficient in medical research. Malawi Med J 2012;24(3):69-71.

12. Ahn SH, Hwang UJ, Jung SH, Kim HA, Kim JH, Kwon OY. Hip external rotator strength and compensatory movement in three different positions. Health 2018;10(1):132-44.

13. Hubbard TJ, Kramer LC, Denegar CR, Hertel J. Correlations among multiple measures of functional and mechanical instability in subjects with chronic ankle instability. J Athl Train
2007;42(3):361-6.

14. Kang MH, Kim GM, Kwon OY, Weon JH, Oh JS, An DH. Relationship between the kinematics of the trunk and lower extremity and performance on the Y-balance test. PM R 2015;7(11):1152-8.

15. Robinson R, Gribble P. Kinematic predictors of performance on the Star Excursion Balance Test. J Sport Rehabil 2008;17(4):347-57. 